\title{
Feedback from the European Bioanalysis Forum: focus workshop on current analysis of immunogenicity: best practices and regulatory hurdles
}

\author{
Joanne Goodman", Simon Cowen², Viswanath Devanarayan³, David Egging ${ }^{4}$, Thomas \\ Emrich$^{5}$, Michaela Golob ${ }^{6}$, Daniel Kramer ${ }^{7}$, Jim McNally ${ }^{8}$, James Munday ${ }^{9}$, Robert \\ Nelson ${ }^{10}$, João A Pedras-Vasconcelos ${ }^{11}$, Timo Piironen ${ }^{12}$, Denise Sickert ${ }^{13}$, Venke Skibeli ${ }^{14}$, \\ Marianne Scheel Fjording ${ }^{15}$ \& Philip Timmerman*,16 \\ ${ }^{1}$ Medlmmune, Aaron Klug Building, Granta Park, Cambridge, Cambridgeshire, CB21 6GH, UK, +44 (0) 2037496244 , +44 (0) \\ 1223471472 \\ ${ }^{2}$ LGC, Queens Road, Teddington, Middlesex TW11 OLY, UK \\ ${ }^{3}$ Charles River Laboratories, 905 Sheehy Dr, Horsham, PA 19044; formerly located at Abbvie during the production of this \\ manuscript \\ ${ }^{4}$ Synthon, Microweg 22, P.O. Box 7071, 6503 GN Nijmegen, The Netherlands \\ ${ }^{5}$ Roche Pharma Research \& Early Development, Roche Innovation Center Munich, Roche Diagnostics GmbH, Nonnenwald 2, \\ 82377 Penzberg, Germany \\ ${ }^{6}$ Nuvisan GmbH, Am Feld 32, 85567 Grafing, Germany \\ ${ }^{7}$ Sanofi Aventis Deutschland GmbH, Industriepark Höchst, D-Frankfurt am Main, Germany \\ ${ }^{8}$ Shire, 300 Shire Way, Lexington MA 02421; formerly located at Merck Serono during production of this manuscript \\ ${ }^{9}$ Covance Laboratories Ltd, Otley Road, Harrogate, HG31PY, United Kingdom \\ ${ }^{10}$ Novimmune SA, 14 Chemin des Aulx, 1228 Plan-les-Ouates, Geneva, Switzerland \\ ${ }^{11}$ US Food and Drug Administration/Center for Drugs Evaluation and Research/Office of Biotechnology Products/Division of \\ Biotech Review and Research 3, MD, USA \\ ${ }^{12}$ Syrinx Bioanalytics, Pansiontie 47, Biohouse D5, 20210 Turku, Finland \\ ${ }^{13}$ Novartis, Kohlenstrasse, WSJ-386.13.05.14, CH-4056 Basel, Switzerland \\ ${ }^{14}$ Norwegian Medicines Agency, Stroemsveien 96, Postboks 6167 Etterstad, 0602 Oslo, Norway \\ ${ }^{15}$ Novo Nordisk A/S, Novo Nordisk Park, DK-2760 Måløv, Denmark \\ ${ }^{16}$ European Bioanalysis Forum, Havenlaan 86c b204, 1000 Brussels, Belgium \\ * Author for correspondence: Tel.: +32 4799101 32; philip@e-b-f.eu
}

\section{European Bioanalysis Forum Workshop, Lisbon, Portugal, September 2016}

At the recent European Bioanalysis Forum Focus Workshop, 'current analysis of immunogenicity: best practices and regulatory hurdles', several important challenges facing the bioanalytical community in relation to immunogenicity assays were discussed through a mixture of presentations and panel sessions. The main areas of focus were the evolving regulatory landscape, challenges of assay interferences from either drug or target, cut-point setting and whether alternative assays can be used to replace neutralizing antibody assays. This workshop report captures discussions and potential solutions and/or recommendations made by the speakers and delegates.

First draft submitted: 23 October 2017; Accepted for publication: 8 December 2017; Published online: 18 January 2018

Keywords: antidrug antibody (ADA) • cut-point • drug tolerance $\bullet$ immunogenicity • neutralizing antibodies $\bullet$ regulatory guidance $\bullet$ target interference

In early 2016 the European Bioanalysis Forum (EBF) [1] created an immunogenicity strategy workstream to address challenges within the bioanalytical community when developing, validating and implementing assays for antidrug antibody (ADA) assessment during biotherapeutic drug development. Just prior to the strategy workstream formation, the European Medicines Agency (EMA) had published a revised draft to the 2006 immunogenicity guideline EMEA/CHMP/BMWP/14327/2006 [2], shortly followed in 2016 by a new draft guidance from the 
US FDA focused on immunogenicity assay development and validation [3]. The EBF community reviewed and commented on both documents during public consultation and it became evident there were several key challenges that warranted further discussion in the format of a focus workshop [4]. This meeting took place in Lisbon in September 2016 with around 80 delegates and 13 speakers.

\section{The current regulatory landscape $\&$ future challenges}

The starting point of the discussions was an overview of the current regulatory landscape given by Michaela Golob (on behalf of the EBF). Globally, there are various regulatory documents concerning immunogenicity, however, guidance documents specifically describing immunogenicity assay development and validation have only been published by the EMA and FDA. Within the International Council for Harmonization (ICH) regions such as Japan and Brazil, there are currently no regulations or guidelines released on this topic. Outside the ICH regions there are several documents delineating immunogenicity but no dedicated regulations describing immunogenicity assay development and validation.

The EBF invited EMA and FDA representatives to present their current draft guidelines and to inform the audience on current discussions and thinking within their agencies. João A. Pedras-Vasconcelos represented the FDA and gave an update on the US perspective on therapeutic protein immunogenicity. He explained regulatory expectations on providing an immunogenicity risk assessment and a suitable sampling plan for clinical studies. As well as the development of validated ADA assays, containing a multi-tiered approach of a binding antibody (screening) assay, a confirmatory assay, a titer assay and potentially a neutralizing antibody (NAb) depending on the stage of development. João referred to preclinical immunogenicity with the primary utility being the interpretation of toxicology and pharmacology data. Nevertheless such preclinical assessment may also reveal potential antibody-related toxicity that could be monitored in clinical trials and therefore appropriate storage of preclinical immunogenicity samples is strongly recommended.

The level of immunogenicity testing within the clinical development program is highly correlated to the potential risk and should be described by a formal risk assessment starting early in development. João then discussed the FDA view on assay design and validation parameters, especially recommending cell-based NAb assays due to their tendency to be more reflective of the in vivo situation. However, competitive ligand-binding assays (CLBAs) might be a suitable alternative in some situations. Additionally, he presented the FDA recommendation on clinical sampling and storage strategy, the latter being of importance should an agency request reanalysis following submission of the Biological License Application.

The EMA was represented by Venke Skibeli who updated the audience on the current status of discussions within the EU. She focused primarily on important updates of the new immunogenicity guideline, focusing on the consequences of an unwanted immune response and asked the community for a proactive approach in verifying the risk to the patient. As immunogenicity issues occur during the entire life cycle of novel protein therapeutics and biosimilars this needs to be considered. Venke explained the scope of the new EMA draft guideline and how the revised document is supplementary to the previous guideline; much of the background information has been removed including some of the general assay concepts, yet they still apply. Nevertheless, this revised EMA guideline now includes the topic of a risk-based approach and how to compile an integrated summary of immunogenicity. Often it is challenging to find data relevant to immunogenicity within dossiers and therefore the integrated summary of immunogenicity should avoid unnecessary questions during filing. Venke also presented an overview on the recommended multi-tiered analytical approach strategy according to the EMA.

\section{A rapidly changing regulatory environment}

Following on from Michaela's introduction to the immunogenicity landscape plus the presentations from the FDA and EMA reviewers, the regulatory theme was continued with further focus on the EMA and FDA draft guidance documents released for public consultation in 2015 and 2016 respectively. The aims of the session were to update delegates on the main comments and suggested revisions submitted by the EBF to both agencies and highlight the key areas of comment. This was followed by a regulatory panel session where questions were posed to the two regulators plus Michaela Golob and Joanne Goodman.

The summary of the EBF comments on the two new draft guidance documents was presented by Joanne (on behalf of the EBF). While a significant number of comments were received, the main focus of the presentation concerned the scope of the documents, molecule formats, biosimilars, positive controls, sensitivity, drug tolerance, specificity/characterization, assay cut-points, NAbs and general comments. While parts of the guidance documents 
are aligned between the two agencies, one of the challenges for the community as a whole is to adhere to both documents to cover multiple filings in different regions. Ideally an ICH harmonization effort dedicated to immunogenicity could be initiated, yet unfortunately this would require at least one more ICH region to produce a guidance document to fulfil the ICH rules.

The definition of a biologic and therefore the need for immunogenicity assessment varied especially in the area of peptides dependent on their size. A risk assessment for any molecule format is vital and multifactorial approaches should be taken when producing the assessment. Additionally, it is recommended that this assessment is formed early in development. However, clinical consequence of the immunogenic response should be the driving factor rather than ADA incidence. Everyone was in agreement that where an endogenous nonredundant counterpart is present, then this should automatically be classed as high risk.

It should be remembered that an immunogenicity assay is only validated when deemed so by regulatory review. This can raise the requirement for further testing and therefore sample storage becomes critical; it is not uncommon for applicants to the FDA, as well as to the EMA, to have to reassess their data or revalidate assays during development. Since it is well known and documented that antibodies are stable in serum and plasma matrices [5], the recommendation was to use trending analysis of positive controls as a viable alternative to formal stability assessments. Validated assays are only required at Biological License Application submission and when supporting a pivotal trial that was generally agreed as Phase III. Although for high-risk molecules this could be as early as Phase I. Otherwise it is acceptable for an assay to only be qualified in the earlier stages of drug development. Furthermore it is usually rare that real-time immunogenicity analysis is required and will only be requested when there is a related safety signal; such cases equate to less than $10 \%$ of FDA submissions.

Finally, NAb assays still need further consideration within the guidance documents, the choice of the assay format is well described but there are no details with respect to the expectation for relative sensitivity and drug tolerance in such assays.

\section{Challenges of drug tolerance \& interferences in immunogenicity assays}

The session on 'challenges of drug tolerance and interferences' included case studies and presentations by James Munday (Covance), Robert Nelson (Novimmune) and Thomas Emrich (Roche Pharma Research and Early Development). This session reviewed the challenges associated with drug tolerance and target interference for immunogenicity assessment assays. James gave an introduction to why ADA assay tolerance and target interference are important to understand and how they can affect data interpretation. He then reviewed strategies that can be applied to improve assay sensitivity when drug tolerance and target interference are an issue. Robert gave two case studies for development programs where target interference and drug tolerance were shown to be an issue for ADA and NAb assays and how these were overcome, and Thomas also gave a presentation on pitfalls in ADA analysis and strategies that can be used to overcome the challenges.

The first presentation given by James Munday described how the existence of biological therapeutics with long acting exposure is resulting in an increased requirement for designing ADA assays with an increased tolerance of free drug. The presentation reviewed methods that can be applied to improve drug tolerance, including optimization of assay design (co-incubation vs stepwise approach), standard acid dissociation as described by Patton et al. [6], solid phase extraction with acid dissociation (Smith et al.) [7], affinity capture elution (Bourdage et al.) [8] and precipitation and acid dissociation (PandA) (Zogbhi et al.) [9]. The review showed how the equipment platform choice, assay design and assay format can all influence the drug tolerance achieved in an ADA assay.

The performance of ADA bridging assays, where the presence of the drug target can cause ADA assay interference, was also reviewed and discussed. A drug target may inhibit ADA binding at the interface between drug and its target epitope resulting in a false negative ADA result. Alternatively, if the target is multimeric it may form a bridge with the critical reagents used in the assay resulting in a false-positive ADA result. Strategies for overcoming such target interference were discussed. These involved either removal of the target by affinity capture/acid dissociationtechniques or by inhibition of the drug-target interaction with the target receptor or with antibodies to a different complementarity-determining region (CDR) to that used by the drug.

Robert Nelson then presented two case studies on 'overcoming drug and target interference in ADA and NAb assays'. The first case study examined challenges in bridging ADA assays when the therapeutic target is soluble and multimeric. Robert showed how antitarget antibodies that are noncompetitive with the monoclonal antibody $(\mathrm{mAb})$ therapeutic had been used in a methodology to deplete the target and so minimize the risk of false-positive 
results in the screening assay. The method included a mild acidic treatment to dissociate drug: ADA complexes and increase drug tolerance and minimize the risk of false-negative screening results.

In the second case study, Robert described how $\mathrm{EC}_{80}$ levels of target and $\mathrm{IC}_{80}$ levels of drug were used to define the experimental window in a cell-based assay to assess the neutralizing activity of ADA, and how the presence of even low levels of target and drug present in samples could confound the NAb assessment. Robert illustrated how a mild acidic treatment was employed to dissociate drug-ADA complexes and denature the soluble target; anti-idiotypic antibodies were then used to remove the $\mathrm{mAb}$ therapeutic and minimize the risk of false negative results. The method included a size exclusion buffer exchange step to remove sample and buffer components, which were incompatible with the cell-based assay.

In his talk, Thomas Emrich emphasized the need for a deep understanding not only of the characteristics of ADAs analytes themselves, but also of their interaction partners, the drug and its target. Using case studies, Thomas presented the impact of assay conditions (incubation time and native/acid pretreatment), the nature of ADA positive controls and to a minor extent different bridging-immunoassay platforms (ELISA and ECLIA) on the analytical sensitivity, especially in the presence of residual drug (drug interference). In another example, it was shown how even low abundant oligomeric soluble targets can mimic ADA responses in a classical bridging ADA assay (target interference) that may lead to false-positive interpretation of ADA testing results. Workarounds by using competitive antitarget antibodies or balanced reduction of sensitivity by sample dilution and benefit from increased drug tolerance/sensitivity due to complex dissociation were presented.

In summary, careful characterization of ADA assays and understanding of the interacting proteins of ADAs under assay conditions is a prerequisite for sensitive and specific detection of ADAs. However, clinically meaningful investigation of immunogenicity is always an integrated analysis of ADA impact on exposure by measurement of pharmacological active drug, clinical safety and efficacy markers.

The session showed that there are a broad array of techniques and assays in the analytical toolbox to build sensitive ADA assays that address the challenges of drug tolerance and target interference. However, to do this the analytical scientist needs a deep understanding of the interacting proteins that are used to build both NAb and ADA assays. It is only then that we can be certain of generating clinically meaningful immunogenicity assessment results. The panel discussion highlighted the challenges faced across the community with building assays that can meet all the requirements but identified ways forward. There was some caution noted on applying acid dissociation techniques to all assays, whether this was needed or not; acid dissociation can change immunogenic epitopes and should therefore only be applied in situations where there is a demonstrated need to increase drug tolerance. Changing the method equilibrium can sometimes be a more effective way of improving assay performance.

\section{Alternatives for NAb assessment}

The aim of the session was to discuss alternatives for detecting clinically relevant NAbs using risk based assessment regarding varying modes of action (MoA) and complexity of biotherapeutics. In addition to evaluating the use of cell-based versus non-cell-based assays, there are ongoing discussions within the biopharmaceutical industry to replace NAb assays with ADA and PK/PD assays, which was highlighted by the first speaker Daniel Kramer (Sanofi). Jim McNally's (Merck Serono) presentation focused on the recent White Paper from the AAPS Working Group on NAbs, which described when to select cell-based versus competitive ligand binding (CLBA) NAb assay formats.

Although there is a strong tendency within the biopharmaceutical industry to replace dedicated NAb assays with an integrated assessment of $\mathrm{ADAs}$ and $\mathrm{PK} / \mathrm{PD}$, regulatory agencies are rather hesitant and still request the standard three-tiered approach (including in vitro NAb assays). Daniel Kramer acknowledged that there are indeed instances in which NAb results do provide data that, but ADA and PK/PD alone, are not sufficient for risk management of patients. This is especially the case if the presence of NAbs is associated with a high safety risk for patients (therapeutic proteins partially or fully identical to a nonredundant endogenous counterpart). However, in most other cases, dedicated in vitro $\mathrm{NAb}$ assays are not really needed to interpret immunogenicity data adequately. Retrospectively this can be seen for example with anti-TNF $\alpha$ mAbs for which the combination of ADA titers, PK and clinical end points were used successfully. Daniel Kramer emphasized that further discussion with authorities is urgently needed in order to gain a common understanding on this topic.

To address the selection of NAb assay format, an AAPS Working Group published a White Paper in 2016 [10] that focused on using an MoA rationale for assay design. Jim McNally presented how the choice between cell-based and non-cell-based formats should be driven by a strong scientific rationale that assesses the neutralization of the 
biotherapeutic's function. Of secondary concern should be the typical parameters associated with development and validation of a bioanalytical assay: sensitivity, tolerance of matrix, drug tolerance and reproducibility.

In practical terms, the assessment of potential assay formats should begin early in the development of a biotherapeutic. The need to generate reagents and identify viable cell lines can require long lead times and, therefore, should be initiated based on a robust immunogenicity risk assessment for the program. In addition, early and frequent consultation with the regulatory agencies can mitigate potential clinical holds and questions around the immunogenicity of the biotherapeutic.

In conclusion, only a minority of safety issues have been detected due to traditional NAb assay findings. Indeed, $\mathrm{ADA}$ and $\mathrm{PK} / \mathrm{PD}$ assays could predict better NAb status in some cases. However, regulatory agencies may not (yet) consider ADA and PK/PD assays as replacements to dedicated NAb assays. In addition, the validation of PD assays can be challenging. Thus, retrospective and prospective analyses are needed to obtain a better understanding of the utility of these assays. In theory, a single PK assay for the determination of active drug may be enough for the determination of NAb status. Further discussions with authorities are needed in order to gain common understanding in this topic. Additionally CLBAs can be used to replace cell-based NAb assays by utilizing a riskbased approach evaluating the MoA and complexity of biotherapeutic treatment. For example, high risk of ADA incidence and low risk of ADA impact may favor CLBAs due to increased throughput and robustness compared with cell-based assays. High risk of ADA impact may place greater emphasis on assay sensitivity in the presence of circulating drug, which may also favor CLBAs. Finally, implementation of the most suitable NAb assay format should always be based on scientific justification. It may be beneficial to develop both CLBA and cell-based NAb assays early during the drug-development process to collect data and gain understanding for the assay format selection.

\section{Cut-point setting in ADA \& NAb assays}

Setting a cut-point is a pivotal part of ADA and NAb assay validations. Incorrect cut-points may lead to failure of establishing relationships between ADA responses and safety or efficacy. The Shankar et al. White Paper [11] describes a statistical approach to cut-point setting during the assay validation and is considered an industry gold standard, but following these recommendations is not always straightforward and may not be considered applicable.

This session provided discussion on different statistical approaches for establishing cut-points, what these mean with respect to expected false-positive rate (FPRs), and practical advice on cut-point setting and outlier removal where biostatistician support may be limited. Case studies were then presented providing practical advice to the challenges of cut-point setting with assay platforms that display limited biological variation and cases where there is a high incidence of pre-existing antibodies.

Statistical aspects of cut-point setting in ADA and NAb assays were covered by two speakers. The first presentation given by Simon Cowen (LGC) focused on the basic concepts, delineating different approaches described in the literature. These vary from classical unbiased estimators of the percentile in question to the use of robust methods and prediction or tolerance limits. Particular emphasis was placed on the underlying statistical question each of these addresses, and the performance of several methods was compared.

A particular discussion point was the recent draft FDA guidance on setting the cut-point, which seeks to control the FPR at the cost of a potentially biased estimate of the relevant percentile [11]. This is a departure from previous guidance. The simulations presented [12] showed that the FPR of the cut-point proposed in the draft of the FDA guidance may yield 10\% FPR on average for the screening assay, which is not congruent with the original intent of the earlier AAPS White Papers [11,13,14], which advocated an average 5\% FPR. However the simulation presented in [12] were based on a sample size of approximately, $\mathrm{n}=50$. When considering the balanced design proposed in [11] that would yield a total of over $\mathrm{n}=300$ sample results ( 50 subjects times three independent runs by each of two analysts), the FPR of the cut-point presented in [12] should be much lower than $10 \%$ on average. Further evaluation using data from several ADA assays has shown the FPR to be approximately $6-7 \%$.

The second speaker, Viswanath Devanarayan (Abbvie), provided a comprehensive practical guide to setting the cut-point that adheres to the requirements laid out previously, which is also straightforward to implement despite the potential complexity of the task. The outlier evaluation process can be tackled using statistical modelling where statistician support is available, or by the simplified approach described here.

Of particular practical importance is the suitability of a prestudy cut-point determined during validation for use during in-study implementation, as the former is an estimate based on a sample with an associated uncertainty. This was investigated by simulation using the Shankar et al. design [11], and it was found that in $95 \%$ of the 
cases the FPR varied between 2 and 11\%. It was recommended that if the in-study baseline samples yielded an FPR outside these limits, a statistical comparison of the means and variances between the prestudy validation data and the in-study predose baseline data should be made to further understand the differences, and a new in-study cut-point should be established.

Additional questions, such as the evaluation of the suitability of negative quality control as a normalization factor for floating cut-point calculations, when and how to use titration cut-points, objective statistical criteria for comparing titer results between samples and criteria for determining treatment-boosted ADA were also discussed.

The session then moved onto two speakers who presented some of the everyday challenges related to cut-point setting. Timo Piironen (Syrinx Bioanalytics) emphasized that to date Shankar et al. [11] has been the only White Paper to provide specific instructions for the validation of immunogenicity assays. This has become a standard approach within the industry and the recent EMA and FDA guidelines follow the same principles. However, the recommendations may be suitable for some assay formats and problematic for others. Very sensitive assays (at or below $\mathrm{ng} / \mathrm{ml}$ range) with a biological background signal close to the instrument background/noise and low variability often do not pass the statistical tests and recommendations of the article. Therefore, robust alternatives for cut-point calculation may be needed. Additionally, sensitivity and selection of the low positive control (LPC) level is often set at an inappropriately low level. For the run control acceptance, the determination of lower limits for positive controls should be adequate, higher limits are not necessary. Furthermore, drug tolerance testing is not meaningful for the LPC sample. This should be performed using a threefold higher level than LPC. For confirmatory assays it may not be possible to inhibit the LPC below the confirmation assay cut-point as the signal has already reached the instrument background level. Thus, the percent inhibition limit should not be applied for the LPC.

During immunogenicity assay validation, especially for human therapeutic antibodies, samples with pre-existing antibodies are usually removed as biological outliers from cut-point analysis. However, with the development of new antibody constructs, for example, nanobodies or single chain fragments there is growing evidence for increased incidence of pre-existing antibodies. Denise Sickert (Novartis) emphasized that immunogenicity cutpoint determination is challenging when a high incidence of pre-existing antibodies is observed, for example, from 20 to up to $100 \%$ of treatment naive subjects. Currently, no standard cut-point evaluation procedure exists for such cases. Therefore, an appropriate assay strategy needs to be established which: distinguishes pre-existing antibodies from false-positive results due to other interfering factors (e.g., multimeric drug target); avoids generation of an inappropriately high cut-point; and reduces the risk of false-negative results. In Denise's presentation three case studies were discussed: usage of immunoglobulin depleted naive sera for cut-point evaluation; immunogenicity sample measurement directly in a confirmatory assay; and selection of pre-existing antibody negative samples assuming a mixed distribution model for cut-point evaluation. Finally it was concluded that the incidence of pre-existing antibodies should be reported along with treatment-induced and treatment-boosted ADA responses. Additionally the impact of pre-existing antibodies on immunogenicity (such as higher immunogenicity incidence in subjects with pre-existing antibodies vs those without) combined with PK, PD, safety and efficiency should be evaluated.

The session wrapped up with a panel discussion that covered a number of topics related to setting of cut-points and practicalities of performing assays.

Of the three types of cut-point that can be calculated following the Shankar et al. design [11]: fixed, floating and dynamic, there was general consensus that a floating cut-point was most desirable for screening assays, and that this method could even be applied in cases where a fixed cut-point was indicated, providing additional reassurance in case of small changes in assay performance during the in-study phase. The use of a dynamic cut-point was strongly discouraged, and was seen as a red flag prompting questions about the robustness of the assay itself.

Discussing the FPR of the screening assay, it was not foreseen that a statistical method yielding a 10\% FPR [12] at the validation stage would provide any practical benefit. Calculation of an FPR at the $5 \%$ level in validation would be expected to yield an in-study FPR of $2-11 \%$. If the in-study baseline samples yielded an FPR outside these limits, this would be a trigger to evaluate an in-study cut-point.

In populations with a relatively high incidence of pre-existing $\mathrm{ADA}$, likely no standard approach is possible. Strategies may include immunoglobulin-depleted drug naive sera for cut-point setting, analysis of all samples in the confirmatory assay using subject specific cut-points, as well as application of titers in determining subject status, although the latter may be difficult in cases of high titers of pre-existing antibodies. Regulators acknowledge these difficulties and advise to interact with them early on a case-by-case basis. 
On the practicalities of performing assays it was noted that three duplicate negative control samples are most often used, spread across the immunoassay plate. Inclusion of a mid-positive control was considered beneficial by some regulators in evaluating the robustness of assay performance when reviewing the validation package, but was not considered a requirement in sample analysis.

\section{Closing panel discussion \& meeting outputs}

The workshop closed with a final discussion where the summaries from each session were presented. There were five tangible outputs from the meeting:

- A need for retrospective and prospective analysis to address whether alternative assay formats such as functional PK assays can be used to replace NAb assays. Such examples should be shared at conferences and via publications with an emphasis on whether immunogenicity safety signals would have been missed if alternative approaches were employed in the place of an NAb assay.

- It is apparent that the industry has not standardized the methods for reporting drug tolerance and that the practical methods differ between laboratories. Additionally antibody titer can be calculated with or without the inclusion of the minimal required dilution; even when minimal required dilution is included in the titer calculation it may range from only the initial sample dilution or may include other sources of dilution such as acid dissociation and/or dilution when added to labelled reagents. Further discussion on this topic is warranted with the aim to standardize these parameters.

- A consortium should be formed to share data and one suggestion was an interaction of the EBF with the European Immunogenicity Platform.

- A focus workshop to discuss case studies where immunogenicity has adversely impacted PK.

- The EBF to consider providing training on immunogenicity, maybe as a preworkshop activity.

\section{Conclusion \& future perspective}

Immunogenicity and appropriate strategies to confidently and accurately assess immune responses in both preclinical and clinical studies is a constantly evolving landscape. New draft guidance documents from both the EMA and FDA have been published and as a result have improved alignment across regulatory regions in some areas of immunogenicity assay development and validation but differences still exist. Harmonization through ICH would be of benefit but not a short-term reality. As further clinical examples are discovered and our understanding deepens, more emphasis on assay performance and characterization of the immune response is requested from regulators. While patient safety is always of upmost concern, the context and relevance of these requests must come from the impact of a resultant clinical response, if indeed one is seen. This should be considered through the generation of a molecule specific risk assessment, preferably at an early stage of drug development. However, there should be a balance of fulfilling regulatory requirements as they appear in the guidance documents and applying sound scientific logic; simply following guidance to the letter will not guarantee a successful drug registration and conversely omitting certain parameters does not automatically mean rejection of the submission. Continued presentation of case studies and discussion within the industry is required and the EBF aim to be an integral part of those activities. For more details please visit the conference website [15].

\section{Acknowledgements}

The authors would like to thank the meeting delegates for their contribution to an interactive and productive workshop.

Financial \& competing interests disclosure

The authors have no relevant affiliations or financial involvement with any organization or entity with a financial interest in or financial conflict with the subject matter or materials discussed in the manuscript. This includes employment, consultancies, honoraria, stock ownership or options, expert testimony, grants or patents received or pending, or royalties.

No writing assistance was utilized in the production of this manuscript. 


\section{References}

1. European Bioanalysis Forum. www.europeanbioanalysisforum.eu/

2. European Medicines Agency. Guideline on Immunogenicity Assessment of Biotechnology-Derived Therapeutic Proteins. EMEA/CHMP/BMWP/14327/2006 Rev. 1.24 September 2015. www.ema.europa.eu/docs/en_GB/document_library/Scientific_guideline/2015/10/WC500194507.pdf

3. US FDA. Assay Development and Validation for Immunogenicity Testing of Therapeutic Protein Products(Draft Guidance) (2016). www.fda.gov/downloads/Drugs/Guidances/UCM192750.pdf

4. $\quad$ EBF Focus Workshop. Current Analysis of Immunogenicity: Best Practices and Regulatory Hurdles. Lisbon, Portugal, 27-28 September 2016.

5. Pihl S, Michaut L, Hendriks J et al. EBF recommendation for stability testing of anti-drug antibodies; lessons learned from anti-vaccine antibody stability studies. Bioanalysis 6(10), 1409-1413 (2014).

6. Patton A, Mullenix MC, Swanson SJ et al. An acid dissociation bridging ELISA for detection of antibodies directed against therapeutic proteins in the presence of antigen. J. Immunol. Methods 304(1-2), 189-195 (2005).

7. Smith HW, Butterfield A, Sun D. Detection of antibodies against therapeutic proteins in the presence of residual therapeutic protein using a solid-phase extraction with acid dissociation (SPEAD) sample treatment prior to ELISA. Regul. Toxicol. Pharmacol. 49(3), 230-237 (2007).

8. Bourdage JS, Cook CA, Farrington DL et al. An affinity capture elution (ACE) assay for detection of anti-drug antibody to monoclonal antibody therapeutics in the presence of high level of drug. J. Immunol. Methods 327(1-2), 10-17 (2007).

9. Zogbhi J, Xu Y, Grabert R et al. A breakthrough novel method to resolve the drug and target interference problem in immunogenicity assays. J. Immunol. Methods 426, 62-69 (2015).

10. Wu B, Chung $\mathrm{S}$, Jiang XR et al. Strategies to determine assay format for the assessment of neutralizing antibody responses to biotherapeutics. AAPS J. 18(6), 1335-1350 (2016).

11. Shankar G, Devanarayan V, Amaravadi et al. Recommendations for the validation of immunoassays used for detection of host antibodies against biotechnology products. J. Pharm. Biomed. Analysis 48, 1267-1281 (2008).

12. Shen M, Dong X, Tsong Y. Statistical evaluation of several methods for cut-point determination of immunogenicity screening assay. $J$. Biopharm. Stat. 25, 269-279 (2015).

13. Mire-Sluis AR, Barrett YC, Devanarayan V et al. Recommendations for the design and optimization of immunoassays used in the detection of host antibodies against biotechnology products. J. Immunol. Methods 289, 1-16 (2004).

14. US Pharmacopeia Chapter <1103. www.drugfuture.com/Pharmacopoeia/usp35/PDF/5678-5686\%20[1103]\%20Immunological\% 20Test\%20Methods\%20-\%20Enzyme-Linked\%20Immunosorbent\%20Assay\%20(ELISA).pdf

15. European Bioanalysis Forum. Focus slides. http://focus201609.europeanbioanalysisforum.eu/slides-2/ 